ARTICLE

Received 10 Mar 2014 | Accepted 1 Dec 2014 | Published 23 Jan 2015

DOI: $10.1038 /$ ncomms7001

OPEN

\title{
Fumarate induces redox-dependent senescence by modifying glutathione metabolism
}

Liang Zheng ${ }^{1}$, Simone Cardaci ${ }^{1}$, Livnat Jerby ${ }^{2}$, Elaine D. MacKenzie ${ }^{1}$, Marco Sciacovelli ${ }^{3}$, T. Isaac Johnson ${ }^{3}$, Edoardo Gaude ${ }^{3}$, Ayala King ${ }^{4}$, Joshua D.G. Leach ${ }^{5}$, RuAngelie Edrada-Ebel ${ }^{6}$, Ann Hedley ${ }^{1}$, Nicholas A. Morrice ${ }^{1}$, Gabriela Kalna1, Karen Blyth ${ }^{1}$, Eytan Ruppin ${ }^{2,7}$, Christian Frezza ${ }^{3, \star} \&$ Eyal Gottlieb ${ }^{1, \star}$

Mutations in the tricarboxylic acid (TCA) cycle enzyme fumarate hydratase (FH) are associated with a highly malignant form of renal cancer. We combined analytical chemistry and metabolic computational modelling to investigate the metabolic implications of $\mathrm{FH}$ loss in immortalized and primary mouse kidney cells. Here, we show that the accumulation of fumarate caused by the inactivation of $\mathrm{FH}$ leads to oxidative stress that is mediated by the formation of succinicGSH, a covalent adduct between fumarate and glutathione. Chronic succination of $\mathrm{GSH}$, caused by the loss of $\mathrm{FH}$, or by exogenous fumarate, leads to persistent oxidative stress and cellular senescence in vitro and in vivo. Importantly, the ablation of p21, a key mediator of senescence, in Fh1-deficient mice resulted in the transformation of benign renal cysts into a hyperplastic lesion, suggesting that fumarate-induced senescence needs to be bypassed for the initiation of renal cancers.

\footnotetext{
${ }^{1}$ Cancer Research UK, Beatson Institute, Switchback Road, Glasgow G61 1BD, UK. ${ }^{2}$ The Blavatnik School of Computer Science-Tel Aviv University, Tel Aviv 69978, Israel. ${ }^{3}$ MRC Cancer Unit, University of Cambridge, Hutchison/MRC Research Centre, Box 197, Cambridge Biomedical Campus, Cambridge CB2 OXZ, UK. ${ }^{4}$ Institute of Cancer Sciences, University of Glasgow, Switchback Road, Glasgow G61 1BD, UK. ${ }^{5}$ School of Veterinary Medicine, College of Medical Veterinary and Life Sciences, University of Glasgow, Bearsden Road, Glasgow G61 1QH, UK. ${ }^{6}$ Strathclyde Institute of Pharmacy and Biomedical Sciences, University of Strathclyde, 16 Richmond Street, Glasgow G1 1XQ, UK. ${ }^{7}$ The Sackler School of Medicine-Tel Aviv University, Tel Aviv 69978, Israel. *These authors contributed equally to this work. Correspondence and requests for materials should be addressed to C.F. (email: cf366@MRC-CU.cam.ac.uk) or to E.G. (email: e.gottlieb@beatson.gla.ac.uk).
} 
F umarate hydratase $(\mathrm{FH})$ mediates the reversible conversion of fumarate to malate in the tricarboxylic acid (TCA) cycle. The biallelic inactivation of $\mathrm{FH}$ leads to hereditary leiomyomatosis and renal cell cancer (HLRCC), a hereditary cancer syndrome characterized by the presence of benign tumours of the skin and uterus, and a highly malignant form of renal cell cancer ${ }^{1}$. Fumarate, which is highly accumulated in FHdeficient cells, has long been considered a major pro-oncogenic factor for HLRCC tumorigenesis ${ }^{2}$. It was initially proposed that the stabilization of the hypoxia-inducible factors (HIFs), caused by the fumarate-dependent inhibition of prolyl hydroxylases, was instrumental for tumour formation 3,4 . However, the recent findings that the genetic deletion of HIFs does not prevent cyst formation in Fh1-deficient mice ${ }^{5}$ challenged their aetiological role in these tumours, indicating that other HIF-independent oncogenic pathways are involved.

Fumarate is a moderately reactive $\alpha, \beta$-unsaturated electrophilic metabolite that can covalently bind to cysteine residues of proteins under physiological conditions. This process, called protein succination, is a feature of FH-deficient tumour tissues, where fumarate accumulates to millimolar levels ${ }^{6}$. Keap1, an E3 ubiquitin ligase and an inhibitor of the transcription factor nuclear erythroid-related factor 2 (NRF2), has recently been identified as a target of protein succination and consequent inactivation, which led to the induction of NRF2 and a potent antioxidant response $e^{5,7}$. Of note, among the most characterized targets of NRF2, haem oxygenase 1 (HMOX1) has been found to be synthetic lethal with $\mathrm{FH}^{8}$ suggesting that the activation of NRF2 and its downstream targets might have an important prosurvival role in Fh1-deficient cancer cells, beyond the control of redox homeostasis.

Although Keap1 succination could explain the antioxidant transcriptional signature in FH-deficient tissues, other direct oxidation mechanisms could contribute to such a response. In fact, the loss of $\mathrm{FH}$ has been previously associated with the increased production of reactive oxygen species $(\mathrm{ROS})^{9}$, and the accumulation of fumarate in FH-deficient cancer cell lines leads to the formation of an adduct between fumarate and glutathione (GSH), which depletes intracellular NADPH and enhances oxidative stress ${ }^{10}$. Nevertheless, a comprehensive investigation of the mechanism by which FH loss triggers oxidative stress, and the biological consequences of such stress, have not been performed.

Systems biology has become an invaluable tool for investigating cellular metabolism as it allows integrating data from different platforms such as transcriptomics and metabolomics. This approach was recently used to identify the molecular determinants of the Warburg effect ${ }^{11}$ and to explore novel therapeutic strategies for cancer such as metabolic synthetic lethality ${ }^{12}$. In the present work, we used a combination of computational biology, metabolomics and mouse genetics to investigate the overall metabolic effects of $\mathrm{FH}$ loss and, in particular, the possible outcomes of FH loss and oxidative stress. We demonstrated that the accumulated fumarate leads to oxidative stress by covalently binding to reduced GSH in a non-enzymatic reaction that forms succinicGSH. We also demonstrated that the oxidative stress induced by GSH succination is necessary and sufficient to elicit cellular senescence in non-transformed cells and that the ablation of senescence can initiate a tumorigenic programme in the kidneys of Fh1-deficient mice.

\section{Results}

Oxidative stress in FH-deficient cells. To elucidate the metabolic changes that occur on the loss of $\mathrm{FH}$, we utilized the previously characterized mouse FH-deficient kidney cells $\left(F h 1^{\Delta / \Delta}\right)$ and their wild-type (wt) isogenic control $\left(F h h^{f l f l}\right)$ (ref. 8). Initially, we found that genes whose expression is upregulated in FH-deficient cells $^{8}$ are significantly enriched with oxidative stress genes (hypergeometric $P$ value of $5.505 \mathrm{e}-04$, see Methods). Reassuringly, genes that are upregulated in HLRCC clinical samples relative to normal kidney tissues ${ }^{7}$ are also significantly enriched with oxidative stress genes (hypergeometric $P$ value of $3.984 \mathrm{e}-06$ ). The genes that featured most highly (Fig. 1a) were then validated by quantitative PCR (qPCR; Fig. 1b). In line with this oxidative stress signature, Fh1-deficient cells exhibit increased ROS production and a substantial drop in GSH/GSSG ratio (Fig. 1c,d).

To better understand the metabolic consequences of FH loss, we performed a comprehensive analysis of extracellular metabolite exchange rates using liquid chromatography-mass spectrometry (LC-MS; Supplementary Table 1). We then generated in silico genome-scale metabolic models of the $\mathrm{FH}$-deficient and wt cells by incorporating the gene expression and metabolite exchange rates measured in these cell lines within a generic metabolic model ${ }^{13}$ (see Methods). Of note, these implemented models not only explore essential reactions for biomass production, as previously done ${ }^{8}$, but also allowed us to predict changes in metabolic fluxes on loss of FH. We first examined the models by testing their ability to predict the measured flux rates via cross-validation (leaving one out). For each iteration, we regenerated a new model based on the gene expression and a partial set of the metabolite uptake and secretion rates and used these models to predict the flux rate through the reaction whose flux measurement was omitted. Significant correlation was found between the measured and predicted flux rates $(R=0.617$ and $0.551, P$ values of $6.59 \mathrm{e}-04$, and $2.63 \mathrm{e}-03$, when testing the wt and FH-deficient models, respectively Supplementary Table 2).

Following the validation of the models, we utilized them to systematically explore the metabolic differences between the FHdeficient and control cells. We computed the capacity of the two models to produce each of the 1,491 metabolites included in the models. The FH-deficient model was found to have a higher capacity to produce 43 metabolites. Among the top ones are four derivatives of GSH (Supplementary Fig. 1a,b). Furthermore, GSH biosynthesis was projected to be an essential metabolic reaction in the Fh1-deficient model (Supplementary Fig. 3b). We also predicted that the FH-deficient model has a significantly lower capacity to produce reducing power, in the form of NADH and NADPH, compared with the wt model (Supplementary Fig. 1c). Of note, in the computational model, the loss of HMOX1, whose essentiality in the Fh1-deficient cells has been previously shown ${ }^{8}$, was expected to exacerbate the decreased ability of the $\mathrm{FH}$ deficient cells to produce NADPH.

Metabolomics revealed GSH succination in FH-deficient cells. To further confirm that GSH metabolism was altered in FHdeficient cells an LC-MS analysis of steady-state intracellular metabolites was performed. Among the most significant metabolites accumulated in FH-deficient cells a putative GSH adduct was detected (annotated by Metlin database as the formate adduct of pyruvilGSH; Fig. 2a). Since this metabolite is specific to FHdeficient cells and is poorly characterized, we decided to elucidate its structure by LC-MS/MS and NMR analyses. The MS/MS fragmentation pattern and the presence of fumarate and GSH fragments as diagnostic daughter ions (Fig. $2 \mathrm{~b}$ ), strongly indicated that this metabolite was wrongly annotated and is instead an adduct of fumarate binding to GSH, which we defined as succinicGSH (Supplementary Fig. 2a for the chemical structure). Furthermore, NMR pulse-field gradient correlation spectroscopy (pfgCOSY), pulse-field gradient total correlation spectroscopy (pfgTOCSY; Supplementary Fig. 2b) and further LC-MS/MS 

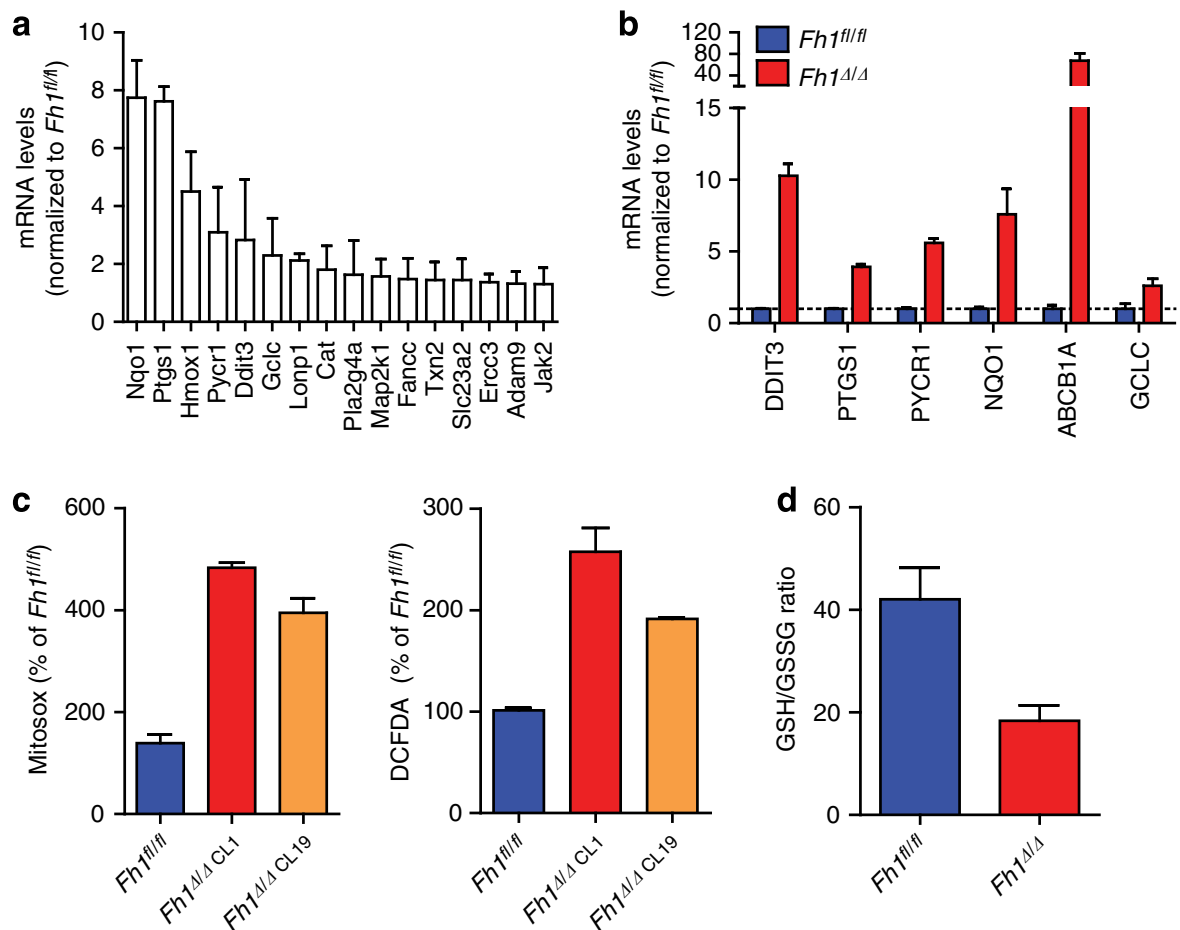

Figure 1 | Antioxidant signature in FH-deficient cells. (a,b) Expression of genes that belong to 'antioxidant response' retrieved from a gene expression array (a) and validated by GPCR (b) from FH-deficient mouse kidney epithelial, as compared with control cells. qPCR data were obtained from three independent cultures and represented as average \pm s.e.m. (c,d) FH-deficient cells exhibit increased oxidative stress measured by mitosox (c), DCFDA (c) and by decreased GSH/GSSG ratio (d). Results were obtained from three independent experiments and represented as average \pm s.e.m.
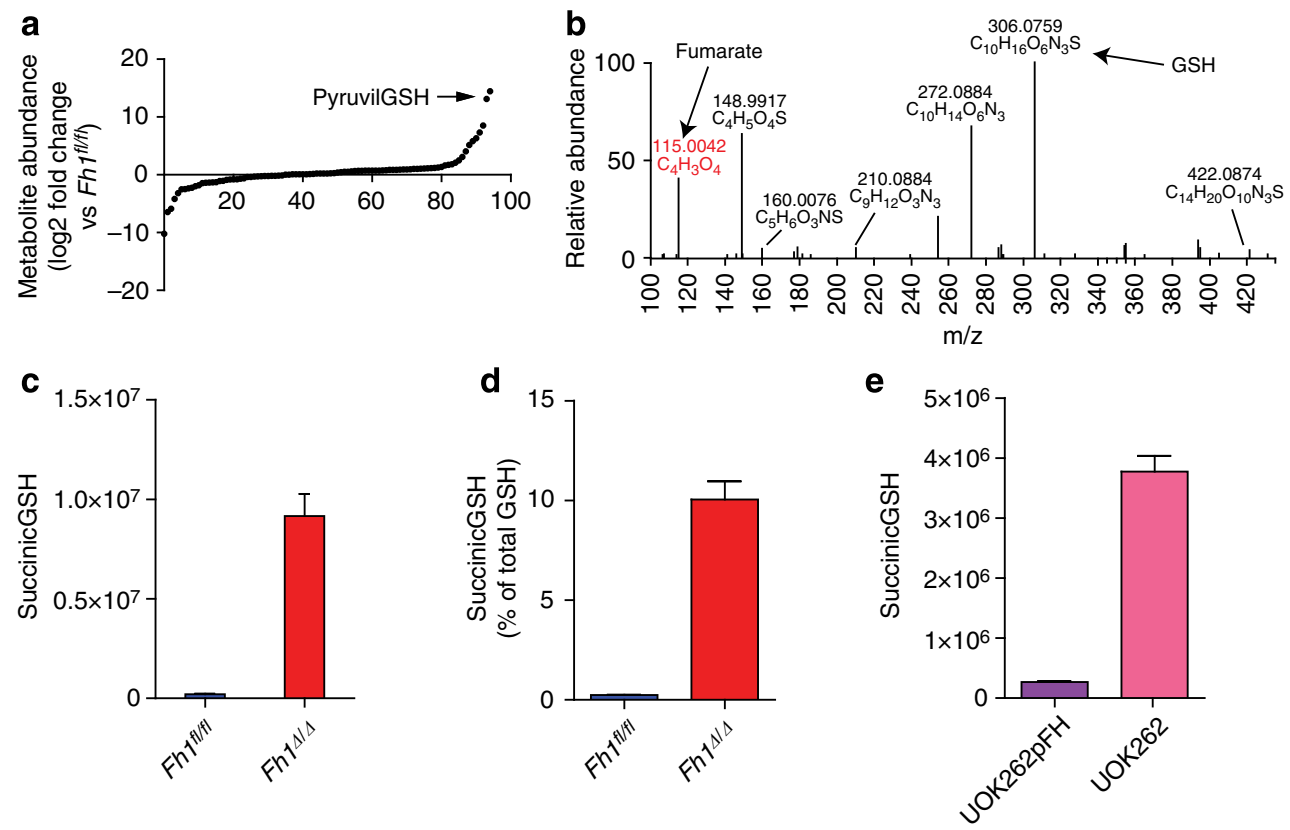

Figure 2 | Accumulation of fumarate leads to GSH succination. (a) Relative abundance of intracellular metabolites in FH-deficient cells measured by LCMS. Results were obtained from nine independent cultures. (b) Fragmentation pattern of the metabolite annotated as pyruvilGSH obtained by LC-MS/MS. (c-e) Intracellular abundance of succinicGSH in mouse (c) and human (e) FH-deficient cells. (d) Amount of succinicGSH compared with the pool of GSH in mouse cells. succinicGSH and GSH were measured by LC-MS. Results were obtained from three independent experiments and represented as average \pm s.e.m.

analysis (Supplementary Fig. 2c) confirmed the chemical structure of this molecule (registered as S-(1,2-Dicarboxyethyl) glutathione: CAS Registry Number [1115-52-2]).
After elucidating the molecular structure of succinicGSH, we confirmed its presence in $F h 1^{\Delta / \Delta}$ cells using targeted LC-MS, showing that succinicGSH represents about $10 \%$ of the total GSH 
in these cells (Fig. 2c,d). It is noteworthy that succinicGSH was also recently characterized in the human FH-deficient renal cancer cell line UOK262 (ref. 10) and we confirmed its presence in these cells (Fig. 2e).

SuccinicGSH is formed by a reaction of fumarate with GSH. To further elaborate on the source of succinicGSH in FH-deficient cells, we tested the hypothesis that this compound is formed by a reaction between accumulated fumarate and GSH. To this end, FH-deficient cells were incubated with uniformly labelled ${ }^{13} \mathrm{C}_{5}$-glutamine, which generates ${ }^{13} \mathrm{C}_{5}$-labelled glutamate and ${ }^{13} \mathrm{C}_{4}$-labelled fumarate, and the isotopologue distribution of succinicGSH was analysed by LC-MS (see Fig. 3a for a schematic diagram of the reactions). SuccinicGSH isotopologues were consistent with carbons coming mostly from fumarate $(m+4)$ and glutamate $(m+5)$, which eventually gave rise to a $m+9$-labelled metabolite (Fig. 3b).

At physiological $\mathrm{pH}$, the thiol residue of GSH is scarcely deprotonated $\left(\mathrm{pK}_{\mathrm{a}}=9.2\right)$. Therefore, the reactions between GSH and electrophilic molecules are generally catalysed by enzymes, such as GSH transferases, which activate GSH by ionizing its thiol group $(\mathrm{SH})$ into the more reactive thiolate $\left(\mathrm{S}^{-}\right)$residue ${ }^{14}$. We therefore wanted to address whether the production of succinicGSH required an enzymatic reaction. To this end, GSH and fumarate, at a concentration found in Fh1-deficient cells ${ }^{15}$, were mixed in a physiological buffer. To avoid interference from endogenous fumarate and succinicGSH from cell extracts, ${ }^{13} \mathrm{C}_{2}$ fumarate was used. When labelled fumarate and GSH were mixed in a physiological buffer, a significant amount of $m+2$ labelled succinicGSH was detected (Fig. 3c). The addition of cell protein extracts from Fh1-deficient cells did not increase and, heat inactivation did not decrease the production of succinicGSH, indicating that the formation of the compound under these conditions is non-enzymatic. Together these results demonstrated that fumarate, when accumulated, leads to GSH succination.

We further investigated the rate of succinicGSH formation and its degradation. To this aim, Fh1-deficient cells were incubated with ${ }^{13} \mathrm{C}_{5}$-glutamine and the kinetics of succinicGSH formation was monitored. The labelling of succinicGSH occurred very rapidly on incubation with labelled glutamine and it tailed GSH biosynthesis (Fig. 3d). Of note, we could not detect the succinated form of the GSH precursor $\gamma$-glutamylcysteine in Fh1-deficient cells, suggesting that GSH, rather than one of its precursors, is subject to succination. We finally investigated the fate of succinicGSH. Experiments on Fh1-deficient cells incubated with ${ }^{13} \mathrm{C}_{5}$-glutamine revealed that succinicGSH is excreted in the media (Fig. 3e) and its isotopologue labelling matches that of intracellular succinicGSH (Fig. 3b). Importantly, in these cells, we have also identified a succinated form of the dipeptide cys-gly, the degradation product of GSH. The rate of production of this metabolite, which followed both GSH and succinicGSH kinetics (Fig. 3f), suggests that succinic cys-gly is likely a breakdown product of succinicGSH. Together this investigation revealed that
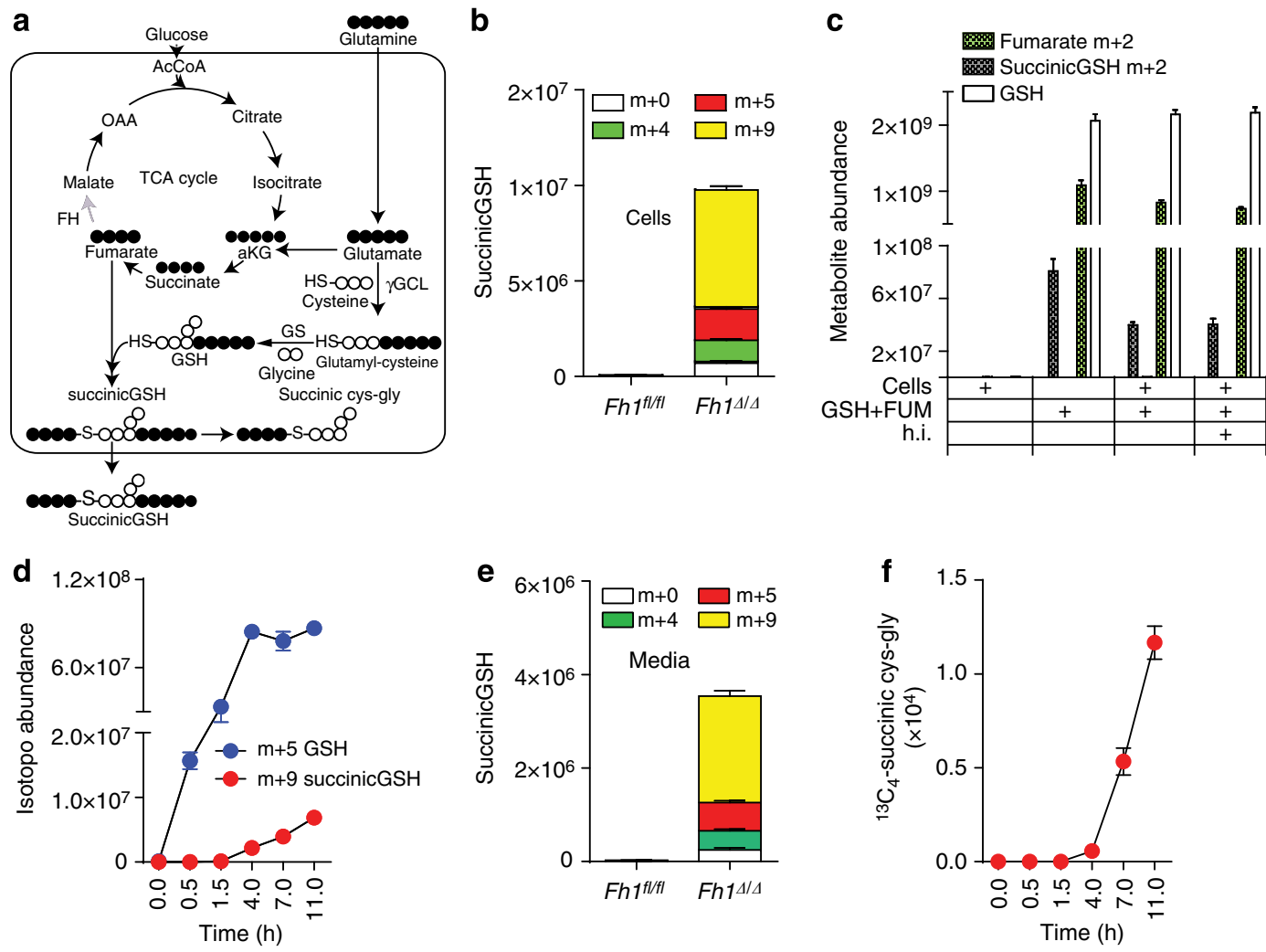

Figure 3 | The formation and fate of succinicGSH. (a) Schematic representation of $\mathrm{GSH}$ biosynthesis in $\mathrm{FH}$-deficient cells on incubation with ${ }^{13} \mathrm{C}_{5}{ }^{-}$ glutamine. The white and black circles under each metabolite illustrate ${ }^{12} \mathrm{C}$ - and ${ }^{13} \mathrm{C}$-, respectively. (b) Isotopologue distribution of succinicGSH was measured by LC-MS after incubating cells of the indicated genotype in the presence of ${ }^{13} \mathrm{C}_{5}$-glutamine for $8 \mathrm{~h}$. (c) GSH and ${ }^{13} \mathrm{C}_{2}$-fumarate were mixed in a physiological buffer in the presence or absence of protein lysates from $\mathrm{FH}$-deficient cells and the amount of ${ }^{13} \mathrm{C}_{2}$-succinicGSH was measured by $\mathrm{LC}$-MS. Where indicated, the cell extracts were heat inactivated (h.i.) before the in vitro reaction. (d) The kinetics of $\mathrm{GSH}_{\text {and }}$ succinicGSH formation from ${ }^{13} \mathrm{C}_{5}{ }^{-}$ Glutamine. (e) The relative level of succinicGSH in the media of mouse cells with the indicated genotype. (f) The kinetics of the formation of the degradation product from GSH was traced by ${ }^{13} \mathrm{C}_{5}$-Glutamine and assessed by LC-MS. Results were obtained from three independent experiments and represented as average \pm s.e.m. 
succinicGSH is formed by succination of newly synthesized GSH and that this metabolite is partly degraded into succinated cys-gly and partly secreted to the media (See Fig. $3 a$ for a schematic of these reactions).

Succination of GSH increases oxidative stress. We wanted to investigate the link between GSH succination and the oxidative stress signature that characterize FH-deficient cells (Fig. 1). Sullivan et al. ${ }^{10}$ previously proposed that enhanced oxidative stress in these cells is caused by the NADPH-depleting conversion of succinicGSH into GSH, a reaction catalysed by GSH reductase (GR). However, this conclusion was based on indirect evidence, that is, consumption of NADPH on incubation of a methylated version of succinicGSH at high concentration and $\mathrm{GR}^{10}$. To test their hypothesis, we measured the activity of GR towards chemically synthesized succinicGSH as compared to its natural substrate oxidized GSH (GSSG) by measuring the products. While GSSG was quickly reduced to GSH, we could not detect GSH production from succinicGSH in the presence of GR (Supplementary Fig. 3a). Therefore, it is unlikely that the detoxification of succinicGSH by GR provides explanation for the enhanced redox stress in Fh1-deficient cells. Several other factors could contribute to the oxidative stress in these cells, including electron transport chain dysfunction, as previously documented $^{16}$. Therefore, to investigate the mechanistic link between fumarate accumulation, succination of GSH, and oxidative stress, respiration-competent Fh1 $1^{f l f l}$ cells were incubated with a cell-permeable derivative of fumarate, dimethylfumarate (DMF). DMF quickly reacted with intracellular GSH leading to the formation of dimethyl-succinicGSH (Fig. 4a). Of note, the incubation with DMF caused a dose-dependent depletion of GSH (Fig. 4b), a substantial drop in the GSH/GSSG ratio (Fig. 4c) and increased oxidative stress (Fig. 4d,e). Furthermore, DMF treatment elicited an antioxidant gene response similar to that observed in $F h 1^{\Delta / \Delta}$ cells (Fig. 4f). Importantly, the incubation with a cell-permeable derivative of GSH, ethyl-GSH, significantly decreased the ROS production triggered by DMF (Fig. 4e). Together these results indicate that the succination of GSH, but not its detoxification, is responsible for the oxidative stress observed in Fh1-deficient cells

GSH succination increases GSH biosynthesis. We hypothesized that the chronic depletion of GSH caused by succination could perturb cell metabolism and contribute to a general deregulation of redox homeostasis. To address this question, we reconstructed the metabolic models previously generated to include GSH succination. Of note, the inclusion of this reaction improved the ability of the FH-deficient model to predict the measured flux rates $(R=0.570, P=1.81 \mathrm{E}-03$; Supplementary Table 2$)$. We found that in the FH-deficient model, but not in the wt model, GSH succination significantly reshaped the generation of reducing power (Supplementary Fig. 1b). Furthermore, the model revealed that GSH succination impinged GSH metabolism and cystine uptake (Supplementary Table 3). Notably, the selective uptake of cystine (an oxidized dimer of cysteine), rather than methionine, as a precursor for cysteine and subsequently GSH biosynthesis imposes a further metabolic constraint: the reduction of cystine to cysteine requires NADPH, the supply of which becomes critical for GSH biosynthesis (see Supplementary Fig. $3 b)$. In line with these predictions, $F h 1^{\Delta / \Delta}$ cells exhibited a significant overexpression of Slc7a11, a member of the cystine transporter $\mathrm{xCT}$, which was functionally associated with increased uptake of cystine (Fig. 5a,b). The rate-limiting step in GSH biosynthesis is the synthesis of $\gamma$-glutamylcysteine
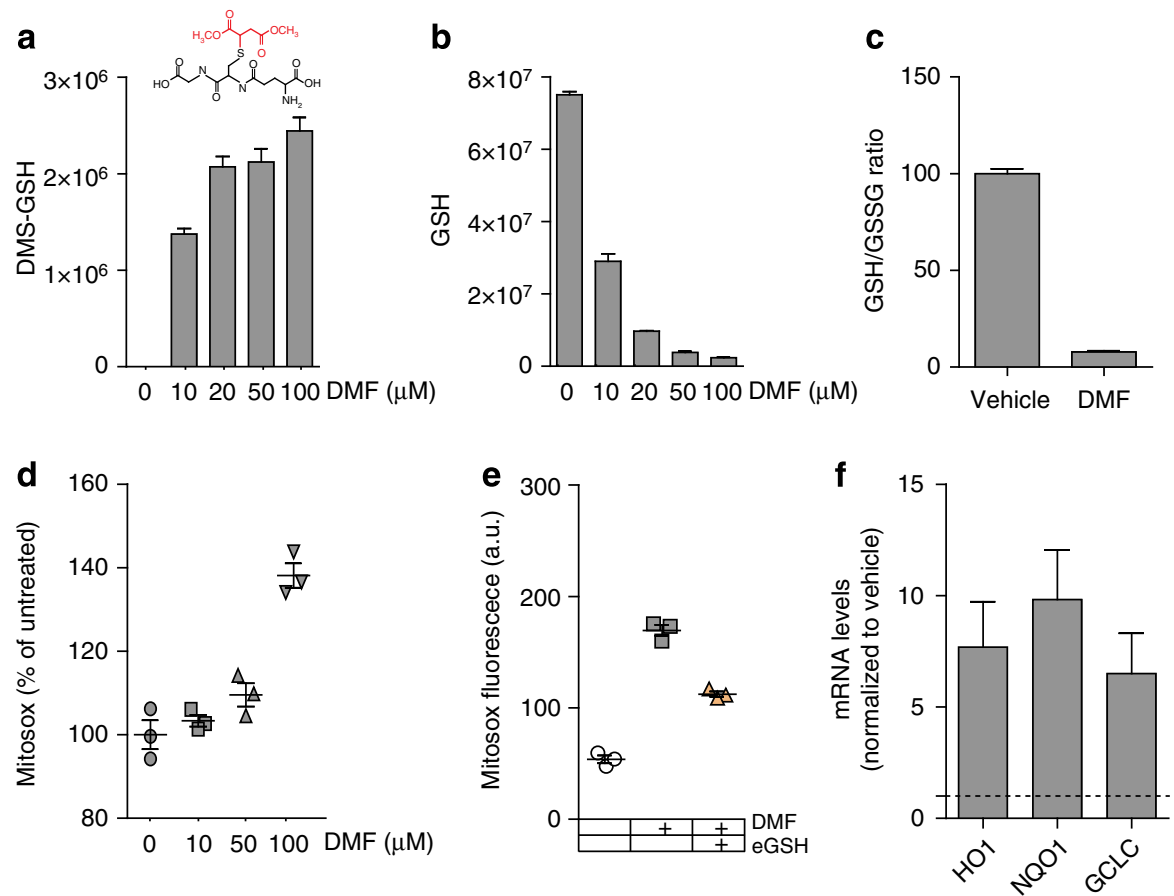

Figure 4 | DMF induces GSH succination and increases oxidative stress. (a-c) Cell-permeable DMF binds to (a) and depletes (b) the intracellular pool of GSH, leading to a decrease in GSH/GSSG ratio (c). The structure of the adduct between GSH and DMF, dimethyl-succinicGSH (DMS-GSH), is indicated in a. (d-f) DMF induces oxidative stress $(\mathbf{d}, \mathbf{e})$ and the activation of an antioxidant response, measured by the expression of antioxidant genes (f). The redox stress caused by DMF is attenuated by incubating cells with a cell-permeable derivative of GSH, ethyl-GSH (e). GCLC, glutamate-cysteine ligase; HO1, haem oxygenase1; NQO1, NAD(P)H dehydrogenase (quinone 1). Results were obtained from three independent experiments and represented as

average \pm s.e.m. 

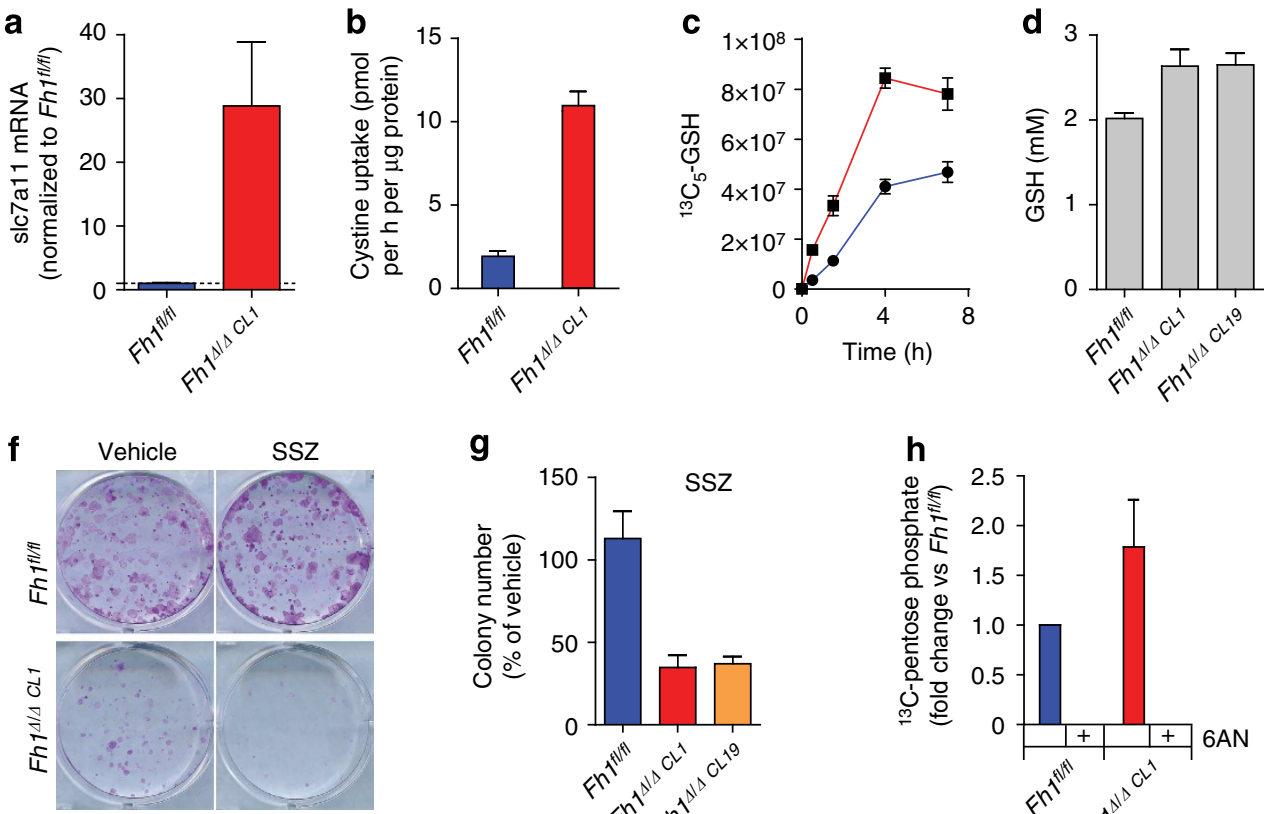

g

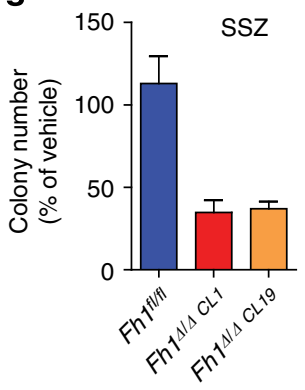

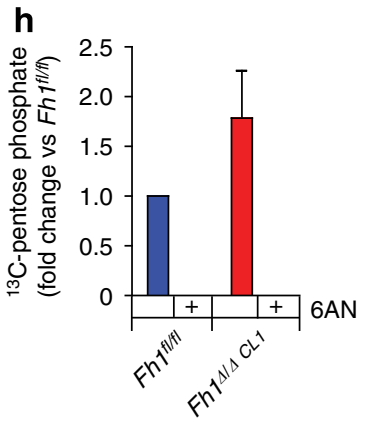

Figure 5 | GSH succination triggers cystine uptake and GSH biosynthesis. (a-c) The loss of FH leads to the upregulation of the cystine transporter (a), increased cystine uptake (b) and accelerated GSH biosynthesis traced from ${ }^{13} \mathrm{C}_{5}$-glutamine (c). (d) The steady-state pool of GSH is increased in $\mathrm{FH}$ deficient cells. (e,f,g) Sulfasalazin (SSZ) inhibits GSH biosynthesis in both wt and FH-deficient cells (e) but selectively impairs cell survival and growth of FH-deficient cells $(\mathbf{f}, \mathbf{g})$. (h,i) 6-Aminonicotinamide (6-AN), an inhibitor of the PPP, inhibits pentose phosphate production in both cell lines as assessed by tracing ${ }^{13} \mathrm{C}_{6}$-Glucose $(\mathbf{h})$, selectively affects the proliferation of $\mathrm{FH}$-deficient cells (i). The images in $\mathbf{f}$ are the representative images of triplicate cultures. Results were obtained from three independent experiments and represented as average \pm s.e.m.

from glutamate and cysteine by $\gamma$-glutamylcysteine synthetase (GCL; see Fig. 3a for a schematic representation of these reactions). GSH is then synthesized by GSH synthetase (GSS), which adds glycine to $\gamma$-glutamylcysteine. Therefore, the GSH biosynthesis rate could be assessed by the rate of incorporation of glutamine-derived glutamate after incubation with ${ }^{13} \mathrm{C}_{5}$-glutamine. The incorporation of glutamate into GSH was faster in FHdeficient cells, meaning that, as predicted by the computational models, GSH production is accelerated in FH-deficient cells (Fig. 5c). Moreover, the increased biosynthesis rate of GSH caused by GSH succination led to a larger steady-state pool of GSH (Fig. 5d). In line with these findings, genes encoding for cystine transport (Slc7a11) and enzymes of the GSH biosynthetic pathway (GSS and GCL), are significantly upregulated in HLRCC patients (Supplementary Fig. 4) ${ }^{7,17}$. All in all, these suggest that increased GSH biosynthesis could be a compensatory mechanism that mitigates the increased oxidative stress in FH-deficient cells.

To test whether GSH biosynthesis is required for the survival of FH-deficient cells, we inhibited cystine uptake using a wellcharacterized xCT inhibitor, sulfasalazine. Reassuringly, the inhibition of cystine uptake significantly impaired GSH biosynthesis in both genotypes (Fig. 5e). However, only $F h 1^{\Delta / \Delta}$ cells were negatively affected by the drug (Fig. 5f). Since GSH biosynthesis requires NADPH supply to reduce cystine to cysteine (Supplementary Fig. 3b), we tested the effects of inhibiting NAPDH production on the survival of FH-deficient cells. The pentose phosphate pathway (PPP) is the major supply of NADPH in cells and so cells were incubated with 6-amino nicotinamide, a known inhibitor of the PPP enzyme 6-phosphogluconate dehydrogenase. 6-Amino nicotinamide effectively halted PPP activity in both genotypes (Fig. $5 \mathrm{~g}$ ) but it only affected the survival of FH-deficient cells (Fig. 5h). In summary, these results indicate that GSH biosynthesis is essential for FH-deficient cells to overcome the oxidative stress caused by GSH succination.
Loss of FH leads to oxidative stress-mediated senescence. We hypothesized that the observed metabolic reprogramming is a required step for the support of cell transformation. Since both the human and mouse FH-deficient cells used here were immortalized cell lines, we tested the effects of GSH succination on primary kidney cells isolated from $F h 1^{f l / f l}$ mouse pups and on the human diploid fibroblasts IMR90. On infection with adenoviral vector expressing Cre recombinase (Ad-Cre), Fh1 fl/fl cells, which lost $\mathrm{FH}$ activity, underwent proliferation arrest, whereas the uninfected cells continued to proliferate (Fig. 6a). The proliferation arrest was associated with a robust induction of the cyclin-dependent kinase inhibitor p21 CIP1/WAF1 (thereafter referred to as p21) and of the cyclin-dependent kinase inhibitor 2A p16 (Fig. 6b). These results suggested that oxidative stressmediated cellular senescence ${ }^{18}$ might be responsible for the observed phenotype. Similarly, the non-transformed nonimmortalized human diploid fibroblast cells IMR90 exhibited a significant upregulation of p21 on acute depletion of FH (Fig. 6c). To confirm the involvement of senescence, we tested the senescence-associated $\beta$-galactosidase (SA- $\beta$-gal) activity in $\mathrm{FH}$ deficient cells. On infection with Ad-Cre, Fh1 $1^{f l / f l}$ exhibited a strong SA- $\beta$-gal staining (Fig. 6d and Supplementary Fig. 5a). Reassuringly, the loss of $\mathrm{FH}$ was also associated with the activation of an antioxidant response similar to that observed in Fh1-deficient cell lines (Supplementary Fig 5b). To ascertain the role of oxidative stress in senescence induction, cells were incubated with ascorbate (vitamin C) or Trolox (vitamin E derivative), two well-characterized antioxidants, which are water or lipid soluble respectively. Importantly, both the growth inhibitory effect, and the induction of SA- $\beta$-gal activity in Fh1deficient primary kidney cells were abrogated by these antioxidants (Fig. 6a,d). These results indicate that redox stress induced by $\mathrm{FH}$ loss is, at least in part, responsible for the ensuing senescence. 

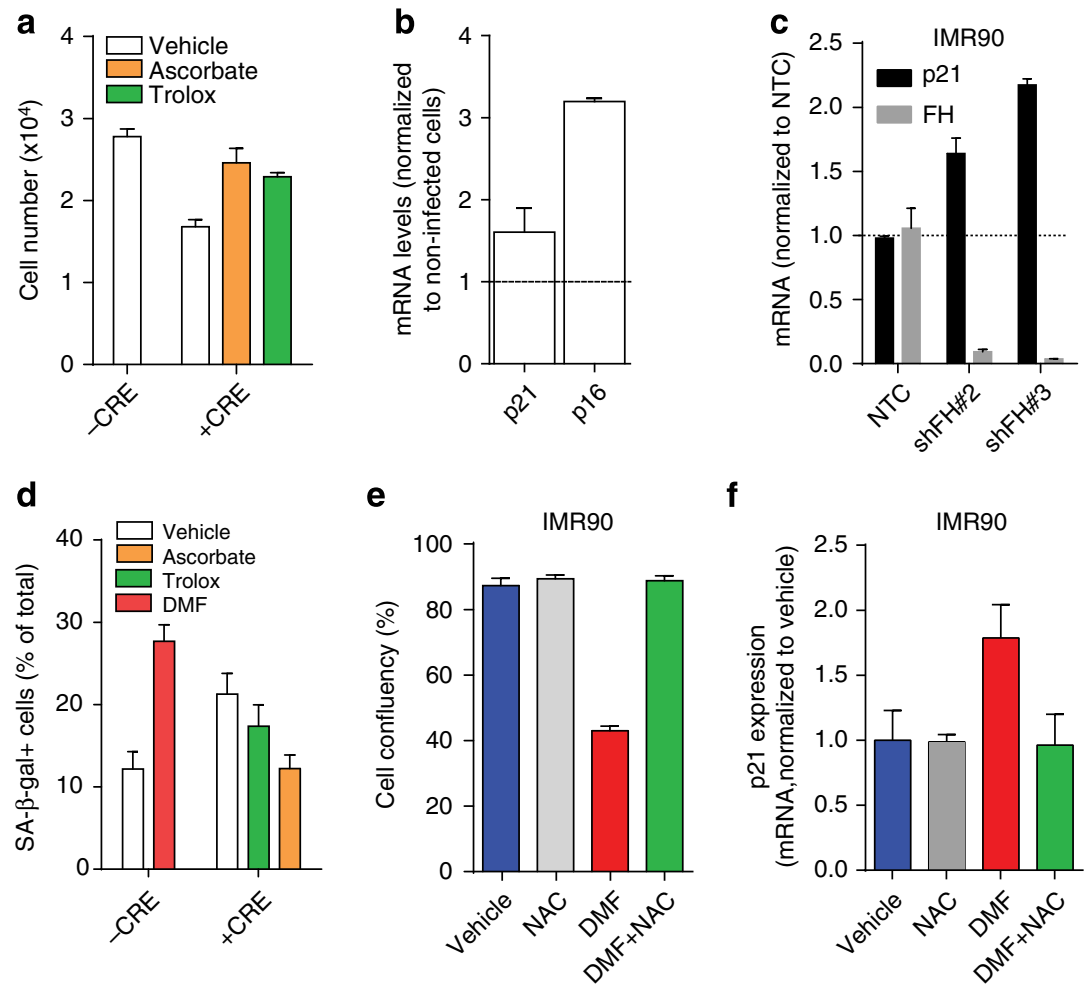

Figure 6 | GSH succination leads to redox stress-induced senescence in primary kidney cells and human diploid fibroblasts. (a) Primary epithelial kidney cells $\left(1 \times 10^{4}\right)$ from $\mathrm{Fh}^{f / / f l}$ animals were either left uninfected or infected with adeno-CRE and treated with vehicle or the antioxidant trolox or ascorbate. Cell number was measured after 2 days of culture. (b) The ablation of FH in cells described in a resulted in the activation of p16 and p21 transcription. Results are expressed as average fold induction over non-infected cells \pm s.e.m (representative experiments; $n=6$ for $p 21$ and $n=3$ for p16). (c) FH and p21 mRNA levels were assessed by qPCR in non-targeting control (NTC)-transfected cells in FH-silenced cells. (d) Microscopy quantitative analysis of senescence-associated $\beta$-galactosidase activity, induced by DMF or adeno-CRE infection, with or without trolox and ascorbate. (e,f) cell growth (e) and p21 mRNA expression levels (f) were assessed in IMR90 cells after DMF treatment, with or without the antioxidant $\mathrm{N}$-acetyl cysteine (NAC). All the results were obtained from three independent cultures and expressed as average \pm s.e.m.

We then wanted to test the direct role of GSH succination in fumarate-induced senescence. Primary kidney cells and IMR90 cells were treated with DMF, a potent inducer of succinationdependent oxidative stress and their proliferation was assessed. DMF elicited a dose-dependent inhibition of cell proliferation and induced SA- $\beta$-gal activity in non-transformed primary mouse kidney epithelial cells (Fig. 6d and Supplementary Fig. 5a,c). Consistent with these results, DMF-treated IMR90 exhibited profound proliferation defects (Fig. 6e and Supplementary Fig. 5d), p21 upregulation (Fig. 6f) and SA- $\beta$ gal positivity (Supplementary Fig. $5 \mathrm{~d}$ ), which were rescued by the incubation with the antioxidant $N$-acetyl cysteine (Fig. 6e,f and Supplementary Fig. 5d,e). These results strongly suggest that fumarate-induced oxidative stress is sufficient for the induction of p21 and the initiation of cell senescence, regardless of cells' genotype.

Fumarate-induced senescence in renal cysts of $F h 1^{\Delta / \Delta}$ mice. Previous work demonstrated that the loss of FH in murine kidneys does not result in overt renal carcinomas, as observed in HLRCC patients, but rather in benign cysts $^{19}$. Our results indicated that the redox stress mediated by the accumulation of fumarate caused by the loss of Fh1 leads to senescence. Therefore, we hypothesized that senescence could be a major adjournment event of the tumorigenic process in Fh1-deficient animals. To test this hypothesis, we used the previously characterized Fh1 ${ }^{f l f l}$ AhCre mice, which develop FH-deficient renal cysts ${ }^{15}$.
Metabolite extraction confirmed the presence of high levels of succinicGSH in the kidneys of these animals (Fig. 7a). In line with our findings in FH-deficient cell lines, many of the epithelial cells facing the lumen of the renal cysts showed an intense staining for p21 (Fig. 7b). To address the role of p21 in the suppression of tumorigenesis in Fh1-null mice, we crossed Fh1 ${ }^{\text {flffl }}$ AhCre with p $21^{-/}$mice, obtaining animals deficient for both Fh1 and p21 in the kidneys $\left(F h 1^{\Delta / \Delta} p 21^{-/-}\right)$. Histological analyses revealed that a simultaneous ablation of these genes resulted in the presence of several hyperproliferative areas in the kidneys. This was assessed by haematoxylin and eosin staining for cellular density and by Ki67 for proliferative capacity of the cells ${ }^{20}$. Strikingly, several areas of the kidneys of $F h 1^{\Delta / \Delta} p 21^{-/-}$animals were characterized by strong Ki67 staining (Fig. $7 \mathrm{c}++$; quantified in Fig. 7d). In fact, 14 out of $24(58.33 \%)$ of the $F h 1^{4 / 4} p 21^{-/-}$animals exhibited dense areas with proliferative and locally invasive kidney epithelial cells (cytokeratin positive) (Fig. 7c ++ and Supplementary Fig. 6). These results strongly suggest that the bypass of senescence may initiate a tumorigenic programme in Fh1-deficient mice.

\section{Discussion}

$\mathrm{FH}$ is emerging as an important mitochondrial tumour suppressor in both hereditary and sporadic forms of cancer ${ }^{2}$. Although FH mutations in HLRCC were discovered more than a decade ago, the specific consequences of the loss of this TCA cycle enzyme remained unclear. The biochemical consequences of Fh1 
a

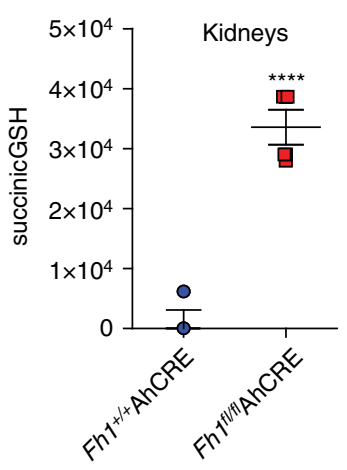

b

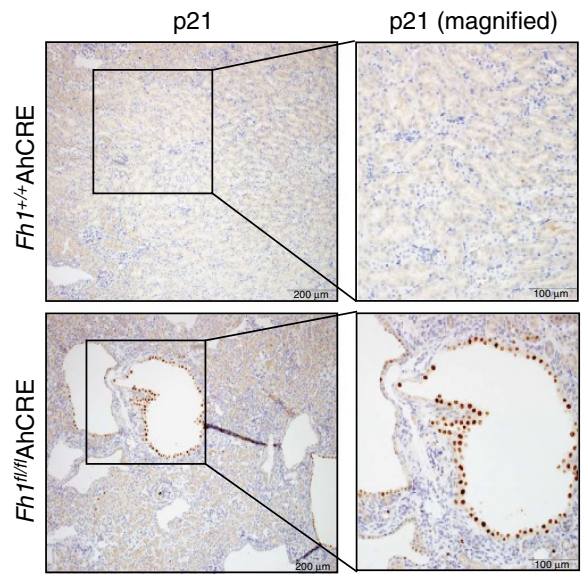

C
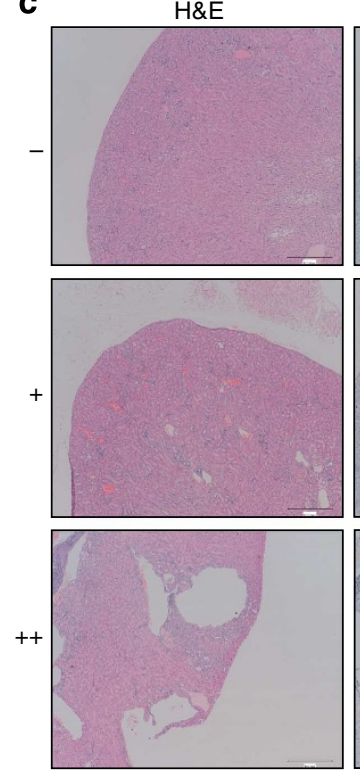

Ki67
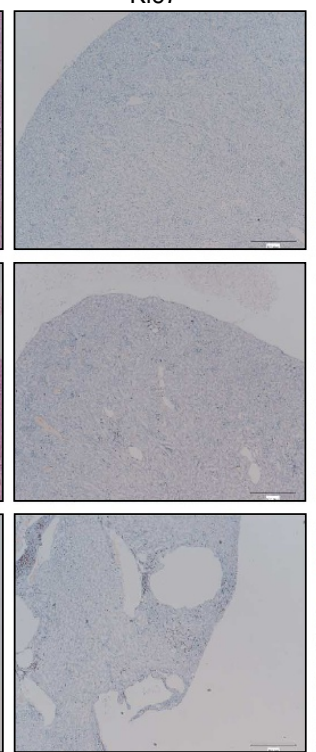

Ki67 (magnified)
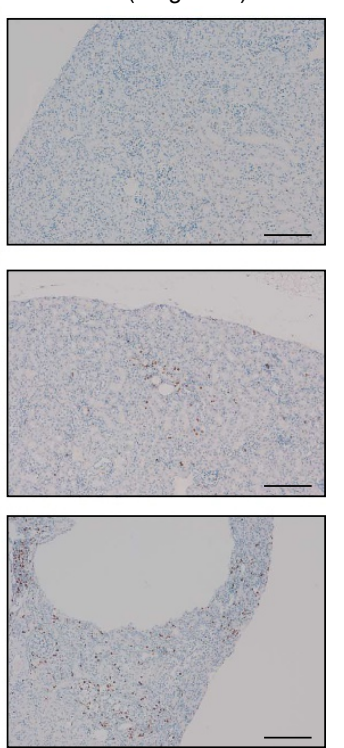

d

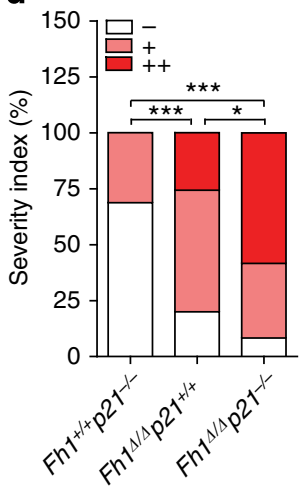

Figure 7 | GSH succination is associated with fumarate-induced senescence in renal cysts. (a) SuccinicGSH was measured in kidney extracts of the indicated animals by LC-MS. Four animals for each genotype were used for this experiment. (b) Representative images of p21 immunostaining performed on dissected kidneys of the indicated mice. (c) Representative haemotoxylin and eosin staining and Ki67 immunostaining images of the different histological grading of kidney hyperplasia observed in 12-18-month-old mice. Representative images from normal kidneys, scored as ( - ), were taken from $\mathrm{Fh}^{+/+} \mathrm{p} 21^{-/-}$animals; kidneys with limited number of cysts (arrows) and areas of hyperplasia with low overall Ki67, staining scored as $(+)$, were taken from Fh1 ${ }^{\Delta / \Delta} \mathrm{p} 21^{+/+}$animals; polycystic kidneys with increased Ki67 immunostaining mainly localized around large cystic areas (arrows), scored as $(++)$, were taken from $\mathrm{Fh}^{\Delta / \Delta} \mathrm{p} 21^{-/-}$. Scale bars represent $500 \mu \mathrm{m}$ and $200 \mu \mathrm{m}$ respectively. (d) Quantitative analysis of the \% distribution of the different hyperplastic grades described in $\mathbf{c}$ in the indicated genotypes. The results are derived from $n=32$ of Fh1 ${ }^{+/+} \mathrm{p} 21^{-/-}, n=35$ of Fh1 ${ }^{\Delta / \Delta} \mathrm{p} 21^{+/+}$ and $n=24$ of $\mathrm{Fh}^{4 / \Delta} \mathrm{p} 21^{-/-}$mice aged 12-18 months. $P$ values calculated by $\chi^{2}$-analysis are indicated $\left({ }^{\star \star \star} P<0.0001,{ }^{\star} P<0.05\right)$.

inactivation in both human and mouse cells have been extensively investigated, revealing a complex pattern of metabolic defects, including deregulation of TCA and urea cycle ${ }^{15}$. Nevertheless, a global analysis of the metabolic rewiring on the loss of Fh1 is still missing. To address this question, we generated in silico models to explore the metabolic consequences of $\mathrm{FH}$ inactivation. These models, which integrate transcriptomics and consumptionrelease metabolomics data to predict metabolic fluxes, revealed unexpected metabolic features of FH-deficient cells. Interestingly, we found that a large number of metabolic pathways became essential on the loss of $\mathrm{FH}$, among which haem biosynthesis and degradation pathway and GSH biosynthesis were the most enriched. While the haem biosynthesis and degradation pathway has already been demonstrated to be synthetic lethal in FH-deficient cells, the role of GSH metabolism in FH-deficient cells has not been investigated before and required further investigation. An untargeted intracellular metabolomics analysis helped to shed light on these findings. We found that the aberrant accumulation of fumarate leads to the persistent depletion of GSH via the formation of the adduct succinicGSH. We confirmed that succinicGSH, which is all but absent from normal cells, accumulates in both human and mouse FH-deficient cells and in the kidneys of Fh1-deficient animals, suggesting that this metabolite could be used as a beacon for toxic accumulation of fumarate. In the work presented here, we showed that succinicGSH is formed by a non-enzymatic reaction between fumarate and the thiol residue of de novo biosynthesised GSH. Furthermore, we found that succinicGSH is secreted by FHdeficient cells and can be degraded, in a process similar to GSH catabolism, into succinic cys-gly.

SuccinicGSH has been recently identified in the human FH-deficient cancer cell line UOK262 (ref. 10). In this work, 
Sullivan et al..$^{10}$ proposed that the detoxification of succinicGSH mediated by GR explains the enhanced oxidative stress in FHdeficient cells. However, our data showed that the contribution of GR to succinicGSH degradation is minor and unlikely to account for increased oxidative stress in these cells. Instead, we found that succination of GSH significantly increases the demands of NADPH to sustain GSH biosynthesis from cysteine. This reaction negatively affects redox homeostasis and, as a compensatory mechanism, FH-deficient cells enhance cystine uptake and GSH biosynthesis. Therefore, although FH-deficient cells experience a chronic oxidative stress, GSH levels are not compromised in these cells. How these cells genetically orchestrate this metabolic reprogramming is not known. The accumulation of fumarate in FH-deficient human cancers has been previously linked with the activation of the transcription factor Nrf2 (refs 5,7), which elicits an antioxidant response and prevents the elevation of ROS to toxic levels in these cells. Keap1 is also regulated by oxidation of its thiol residues ${ }^{21}$; therefore, the increased oxidative stress observed in FH-deficient cells could also contribute to Nrf2 activation. We hypothesize that both the redox stress caused by GSH succination and Keap1 succination contribute to the emergence of the antioxidant signature and Nrf2 activation to support GSH biosynthesis and to maintain a viable level of oxidative stress.

Persistent oxidative stress has been previously linked to the induction of senescence ${ }^{18}$. We reasoned that overt oxidative stress caused by the loss of FH in non-transformed cells could instigate a tumour suppressive mechanism that could explain why Fh1deficient animals do not develop carcinomas but only benign cysts $^{19}$. To accurately dissect the effects of accumulation of fumarate in early phases of epithelial transformation, we silenced $\mathrm{FH}$ in non-immortalized non-transformed cells. In primary epithelial kidney cells and in human diploid fibroblasts, the loss of FH or incubation with DMF leads to a robust induction of senescence, confirmed by proliferative arrest, p21 upregulation and SA- $\beta$-galactosidase activity. The incubation with antioxidants abrogated the anti-proliferative response, suggesting that the oxidative stress induced by fumarate accumulation is responsible for the induction of senescence. In line with these results, we observed that some renal cysts exhibit a strong positive staining for $\mathrm{p} 21$, indicating that senescence is also a factor that may prevent or delay malignant transformation in vivo. In line with this notion, the co-ablation of p21 and Fh1 resulted in high proportion of hyperprolifearative areas in the kidney. Importantly, p21, but not p16 induction is also observed in human HLRCC patients (Supplementary Fig. 4). These results confirm the association between FH loss and p21 induction in human tumours. Furthermore, they may indicate that in human FH-deficient renal cancer, the lack of p16 induction may help avoiding the senescence phenotype.

The observation that the loss of $\mathrm{FH}$, a bona fide tumour suppressor gene ${ }^{22}$, could lead to senescence, although counterintuitive, suggests that the role of $\mathrm{FH}$ in tumorigenesis is complex and might involve secondary oncogenic alterations to overcome senescence. It is worth mentioning that the loss of FH is not the only example of an oncogenic event that leads to senescence at early stages of tumorigenesis. Other examples include the loss of the tumour suppressors VHL and PTEN ${ }^{23,24}$ as well as the activation of the oncogenes Ras or $\mathrm{BRAF}^{25-27}$. Although the underlying mechanisms that lead to senescence within these models might be different, a general accepted paradigm is that the unrestrained proliferation elicited by an oncogenic event leads to the activation of tumour restraining events. In this work we demonstrated that in the case of FH loss, this activity is mediated by fumarate, at least in part via GSH succination.

\section{Methods}

Animal work. Animal work was carried out with ethical approval from University of Glasgow under the Animal (Scientific Procedures) Act 1986 and the EU Directive 2010 (PPL 60/4181). Animals were housed in individual ventilated cages in a barrier facility proactive in environmental enrichment. AhCre and $p 21^{-1-}$ $\left(C d k n 1 a^{t m 1 L e d}\right)$ mice were sourced from Owen Sansom (Cancer Research UK, Beatson Institute, Glasgow $)^{28,29}, F h^{f l / f l}$ mice kindly provided by Iain Tomlinson (University of Oxford) ${ }^{19}$ and all were maintained on a C57Bl6 background. Experimental mice (mixed sex) were aged to $12-18$ months and no difference between male and female animals was observed.

Cell culture. $F h 1^{f l / f l}, F h 1^{\Delta / \Delta}$, UOK262 and UOK262pFH cell lines were obtained and cultured as previously described ${ }^{8}$. In brief, all cell lines were cultured in DMEM supplemented with $10 \%$ fetal bovine serum and $2 \mathrm{mM}$ glutamine. The mouse cell lines were also supplemented with $1 \mathrm{mM}$ pyruvate and $50 \mu \mathrm{g} \mathrm{ml}^{-1}$ uridine.

Immunohistochemistry. Mouse kidneys were fixed in $10 \%$ neutral buffered formalin and paraffin embedded. Sections $(4 \mu \mathrm{m})$ were cut and stained for Ki67 (1:100 Thermo RM-9106), Cytokeratin (Cytokeratin, Pan Ab-1, Mouse Monoclonal Antibody 1:100, Thermo, MS-34) and p21 (1:500 Santa Cruz, sc471).

Chemical reagents. Unless otherwise stated, all chemical compounds were purchased form Sigma (Gillingham, Dorset, UK). ${ }^{13} \mathrm{C}$-labelled compounds were purchased by Cambridge Isotopoes (Tewksbury, MA).

The transcriptomics signature of FH-deficient cells. To examine whether the $F h 1^{\Delta / \Delta}$ cells are under oxidative stress based on their gene expression (http:// www.ncbi.nlm.nih.gov/geo/query/acc.cgi?acc=GSE63438) we identified genes whose expression is significantly higher in the $F h 1^{\Delta / \Delta}$ cells compared with the $F h 1^{f l / f l}$ cells (false discovery rate-corrected $P$-values $<0.05$ ) (ref. 8). We then assembled a set of 281 genes that are known to be involved in the response to oxidative stress. This set includes genes that are annotated with one of the 32 gene ontology terms which are child terms of the gene ontology term response to oxidative stress ${ }^{30}$ (for example, response to ROS, response to superoxide, response to oxygen radical). We examined if the genes that are significantly upregulated in the $F h 1^{\Delta 4 \Delta}$ cells compared with the $F h 1^{f l / f l}$ cells are enriched with this set of oxidative stress genes, via a hypergeometric enrichment test. We then examined if $\mathrm{FH}$ deficiency induces a gene expression signature of oxidative stress also in clinical samples. To this end, we analysed a previously defined set of 1,397 genes that are upregulated in HLRCC relative to normal kidney tissues ${ }^{7}$, and tested if this set is enriched with oxidative stress genes by performing a hypergeometric enrichment test.

Metabolomic extraction of mouse tissue. Kidneys were excised and immediately processed for metabolomics extraction. Equal amounts of renal tissue were lysed in $250 \mu \mathrm{l}$ of extraction solution per each $10 \mathrm{mg}$ of tissue in Precellysis vials following manufacturer's instruction. The extraction solution contained $30 \%$ acetonitrile, $50 \%$ methanol and $20 \%$ water. The suspension thus generated was immediately centrifuged at $16,000 \mathrm{~g}$ for $15 \mathrm{~min}$ at $0^{\circ} \mathrm{C}$. The supernatant was then submitted to LC-MS metabolomic analysis.

Metabolomic extraction of cell lines. Cells $\left(5 \times 10^{5}\right)$ were plated onto six-well plates and cultured in standard medium for $24 \mathrm{~h}$. For the intracellular metabolomic analysis, cells were quickly washed for three times with PBS to remove contaminations from the media. The PBS was thoroughly aspirated and cells were lysed by adding a precooled ES. The cell number was counted in a parallel control dish, and cells were lysed in $1 \mathrm{ml}$ of ES per $1 \times 10^{6}$ cells. The cell lysates were vortexed for $5 \mathrm{~min}$ at $4^{\circ} \mathrm{C}$ and immediately centrifuged at $16,000 \mathrm{~g}$ for $15 \mathrm{~min}$ at $0{ }^{\circ} \mathrm{C}$. The supernatants were collected and analysed by LC-MS. For the metabolomic extraction of spent media, $50 \mu \mathrm{l}$ of cell media were deproteinized by 1:16 dilution in a solution composed of $80 \%$ acetonitrile and $20 \%$ water. The supernatants were then processed as described above. Fresh medium without cells was incubated in the same experimental conditions and used as a reference.

LC-MS metabolomic analysis. For the LC separation, column A was the Sequant Zic-Hilic $(150 \mathrm{~mm} \times 4.6 \mathrm{~mm}$, internal diameter (i.d.) $5 \mu \mathrm{m})$ with a guard column $(20 \mathrm{~mm} \times 2.1 \mathrm{~mm}$ i.d. $5 \mu \mathrm{m})$ from HiChrom, Reading, UK. Mobile phase A: $0.1 \%$ formic acid v/v in water. Mobile B: $0.1 \%$ formic acid v/v in acetonitrile. The flow rate was kept at $300 \mu \mathrm{lmin}^{-1}$ and gradient was as follows: 0 min $80 \%$ of B, $12 \mathrm{~min}$ $50 \%$ of B, $26 \min 50 \%$ of B, $28 \min 20 \%$ of B, $36 \min 20 \%$ of B, $37-45 \min 80 \%$ of B Column B was the sequant Zic-pHilic $(150 \mathrm{~mm} \times 2.1 \mathrm{~mm}$ i.d. $5 \mu \mathrm{m})$ with the guard column $(20 \mathrm{~mm} \times 2.1 \mathrm{~mm}$ i.d. $5 \mu \mathrm{m})$ from HiChrom, Reading, UK. Mobile phase C: $20 \mathrm{mM}$ ammonium carbonate plus $0.1 \%$ ammonia hydroxide in water. Mobile phase D: acetonitrile. The flow rate was kept at $100 \mu \mathrm{min}^{-1}$ and gradient as follow: $0 \mathrm{~min} 80 \%$ of D, $30 \mathrm{~min} 20 \%$ of D, $31 \mathrm{~min} 80 \%$ of D, $45 \mathrm{~min} 80 \%$ of D The mass spectrometer (Thermo Exactive Orbitrap) was operated in a polarity switching mode. 
${ }^{13} \mathrm{C}$-glutamine and cystine labelling experiments. Cells $\left(5 \times 10^{5}\right)$ were plated onto six-well plates and cultured in standard medium for $12 \mathrm{~h}$. The medium was then replaced by fresh medium supplemented with either $2 \mathrm{mM} \mathrm{U}-{ }^{13} \mathrm{C}$-Glutamine or $0.2 \mathrm{mM}^{13} \mathrm{C}_{2}$-Cystine for the indicated time.

Orbitrap-Velos $\mathbf{M S}^{2}$ fragmentation. The S-(1, 2-Dicarboxyethyl) GSH standard (H-1556.1000, Bachem) was dissolved in water and diluted to $40 \mu \mathrm{g} \mathrm{ml}^{-1}$ with a $50 \%$ mixture of acetonitrile and water. An Orbitrap-Velos mass spectrometer was used in the negative mode, with HCD fragmentation $\mathrm{MS}^{2}$ energy of 50 unit.

NMR measurement. Proton and two-dimensional pfgCOSY as well as pfgTOCSY were measured for standards and samples on an ECX 400 Jeol NMR equipped with a pulse-field gradient autotune $40 \mathrm{TH} 5 \mathrm{AT} / \mathrm{FG}$ broadband high-sensitivity probe. ${ }^{1} \mathrm{H}$ NMR (400 MHz, Methanol-d4) $\delta 3.98(\mathrm{~s}, 2 \mathrm{H}), 3.85-3.71(\mathrm{~m}, 2 \mathrm{H}), 3.38(\mathrm{~s}, 1 \mathrm{H}), 2.97$ $(\mathrm{dq}, J=18.1,9.3 \mathrm{~Hz}, 3 \mathrm{H}), 2.75$ (dd, $J=17.1,5.9 \mathrm{~Hz}, 3 \mathrm{H}), 2.72-2.58(\mathrm{~m}, 1 \mathrm{H}), 2.22$ $(\mathrm{m}, 1 \mathrm{H})$.

Bioinformatics processing and statistical analysis of the metabolomic data. The metabolomics analyses obtained from cells and media extractions were $\log 2$ transformed and the replicates normalized using quantile normalization. Then, a multi-factor analysis of variance and multiple test correction were used to identify metabolites significantly different between experimental groups. Analyses were performed using Partek Genomics Suite Software, version 6.5 Copyright 2010 and $\mathrm{R}$ version 2.14.0.

In vitro succination reaction. $\mathrm{GSH}(2 \mathrm{mM})$ and $8 \mathrm{mM}^{13} \mathrm{C}_{2}$-fumarate were mixed in $2 \mathrm{ml}$ of a solution composed of $50 \mathrm{mM} \mathrm{NaCl}, 20 \mathrm{mM}$ Tris and $0.1 \%$ Triton-X$100, \mathrm{pH}=7.4,4^{\circ} \mathrm{C}$ for $8 \mathrm{~h}$ in the presence or absence of cell lysate obtained from $2 \times 10^{6} \mathrm{Fh} 1^{4 / 4}$ cells previously heat inactivated $\left(100^{\circ} \mathrm{C}\right.$ for $\left.1 \mathrm{~h}\right)$ or left untreated. Protease inhibitor cocktail (Sigma) was used following manufacturer's instructions. At the end of the experiment, the mixture was quenched using the extraction solution for metabolomics (see above) and the extracts were spun down at maximum speed for $10 \mathrm{~min}$. The supernatant was subsequently analysed by LC-MS.

DMF treatment. Cells $\left(5 \times 10^{5}\right)$ were plated onto six-well plates and cultured in standard medium for $24 \mathrm{~h}$. Then the cell culture medium was replaced with medium supplemented with the indicated concentrations of DMF for the indicated time. Cells were then harvested and extracted as described above.

GSH GR assay. SuccinicGSH ( $0.2 \mathrm{mM}$, purchased from BACHEM) and GSSG $(2 \mathrm{mM})$ were separately dissolved in a solution containing $50 \mathrm{mM}$ Tris and $5 \mathrm{mM}$ EDTA. The $\mathrm{pH}$ was adjusted at 7.8 to mimic the $\mathrm{pH}$ of the mitochondrial matrix. Substrates were added to a solution of $250 \mu \mathrm{M}$ NADPH and $0.05 \mathrm{U} \mathrm{ml}^{-1} \mathrm{GSH}$ GR. Metabolite production was monitored by LC-MS.

qPCR experiments. mRNA was extracted as previously described ${ }^{8}$. In brief, RNA was extracted from cells of the indicated genotype using the Qiagen RNAeasy RNA extraction kit (Qiagen, Manchester, UK) following the manufacturer's instructions.mRNA $(1 \mu \mathrm{g})$ was retrotranscribed into cDNA using High Capacity RNA-to-cDNA kit (Life Technologies, Paisley, UK). qPCR reaction was performed using $200 \mathrm{ng}$ of cDNA, the indicated primers and fast Sybr green mastermix (Life Technologies). Primers were designed using ROCHE universal probe library primer design tool (version 2.45 and 2.48) and the sequences were as follow:

p21 FW: 5'-GAGGGCTAAGGCCGAAGATG-3', RV: 5'-GCAGACCAGCCTG ACAGATTTC- 3

Rpl34 FW: 5'-GTGAGGGACGAGGAGAGGTG-3', RV: 5'-ACCATCCTGAG TGCCGCTTC- $3^{\prime}$

Slc7al1 FW: 5'-TGGGTGGAACTGCTCGTAAT-3', RV: 5'-AGGATGTAGCG TCCAAATGC- $3^{\prime}$

DDIT3 FW: 5-GCGACAGAGCCAGAATAACA-3', RV 5'-GATGCACTTCCT TCTGGAACA- $3^{\prime}$

PTGS1 FV: 5'-CCTCTTTCCAGGAGCTCACA-3', RV: 5'-TCGATGTCACCG TACAGCTC- $3^{\prime}$

PYCR1 FW: $5^{\prime}$-CTGACCATGTCAAGCCACTG- ${ }^{\prime}$, RV $5^{\prime}$-TTCCCAGGGATA GCTGAGGT- $3^{\prime}$

NQO1 FW: 5' - AGCGTTCGGTATTACGATCC-3', RV: 5' - AGTACAATCAG GGCTCTTCTCG-3'

ABCB1A FW: 5'-GGGCATTTACTTCAAACTTGTCA-3', RV: 5'-TTTACAA GCTTCATTTCCTAATTCAA-3

GCLC FW: 5'-ATGATAGAACACGGGAGGAGAG-3', RV: 5'-TGATCCTAA AGCGATTGTTCTTC- $3^{\prime}$

HMOX1 FW: $5^{\prime}$-gtcaagcacagggtgacaga- $3^{\prime}$ RV $5^{\prime}$-atcacctgcagctcctcaaa- $3^{\prime}$

For the qPCR reactions, $0.5 \mu \mathrm{M}$ primers were used $-1 \mu \mathrm{l}$ of Fast Sybr green gene expression master mix; $1 \mu \mathrm{l}$ of each primers and a $4 \mu \mathrm{l}$ of a 1:10 dilution of cDNA in a final volume of $20 \mu \mathrm{l}$ were used. Real-time PCR was performed in the 7500 Fast Real-Time PCR System (Life Technologies) using the fast Sybrgreen programme and expression levels of the indicated genes were calculated using the $\Delta \Delta \mathrm{Ct}$ method by the appropriate function of the software using actin as calibrator.

Primary kidney cells isolation and infection. Primary kidney epithelial cells were isolated from $F h 1^{+/+}$and $F h^{f l / f l} \mathrm{p} 5$ mice as described previously ${ }^{31}$ and cultured in DMEM supplemented with $10 \%$ fetal bovine serum, $2 \mathrm{mM}$ glutamine and $1 \mathrm{mmoll}^{-1}$ pyruvate. Cells were infected with Cre recombinase-encoding adenovirus (Ad5-CMV-Cre-GFP, Vector Development Laboratory) using 200 p.f.u. per cell. After 2 days, cells were transferred on 24-well plates for growth curve determination and SA- $\beta-$ Gal Staining.

Lentiviral infection of IMR90. The viral supernatant for infection was obtained from the filtered growth media of the packaging cells HEK293T transfected with $3 \mu \mathrm{g}$ psPAX, $1 \mu \mathrm{g}$ pVSVG, $4 \mu \mathrm{g}$ of short hairpin RNA (shRNA) constructs and $24 \mu \mathrm{l}$ of Lipofectamine 2000 (Life Technology). Cells $\left(3 \times 10^{5}\right)$ were then plated on sixwell plates and infected with the viral supernatant in the presence of $4 \mu \mathrm{g} \mathrm{ml}^{-1}$ polybrene. After two days, the medium was replaced with selection medium containing $1 \mu \mathrm{g} \cdot \mathrm{ml}^{-1}$ puromycin. The expression of the shRNA constructs was induced by incubating cells with $2 \mu \mathrm{g} \mathrm{ml}^{-1}$ doxycyclin. The shRNA sequences were purchased from Thermoscientific (NTC: \# RHS4743; sh \#2 V3THS_324847 and sh\#3: V3THS_324846)

Senescence-associated (SA)- $\boldsymbol{\beta}$-Gal staining. For mouse epithelial kidney cells, $4 \times 10^{4}$ cells were treated for 2 days with $50 \mu \mathrm{M}$ DMF and a Senescence $\beta$ Galactosidase Staining Kit (Cell Signaling Technology) was used. The staining was performed according to manufacturer's instructions and images were acquired with a Zeiss Axiovert $200 \mathrm{M}$ microscope. To quantify SA- $\beta$-gal positive cells, cells were counted in four random fields in each of the triplicates wells.

For IMR90, $4 \times 10^{4}$ cells were plated onto coverslips in a 24 -well plate. The day after the medium was replaced with medium containing either DMF or $N$-acetyl cysteine or a combination of the two drugs. The medium was replenished every day for a total of 5 days of treatment. At the end of the experiment, cells were washed in PBS and fixed with $0.5 \%$ glutaraldehyde solution (in PBS) for $15 \mathrm{~min}$ at room temperature. Immediately after cells were washed twice with $1 \mathrm{mM} \mathrm{MgCl}$ in PBS (pH 6.0) and incubated with a staining solution composed of $1 \mathrm{mg} \mathrm{ml}^{-1}$ 5 -Bromo-4-chloro-3-indolyl- $\beta$ - D-galactopyranoside (prepared in $\mathrm{N}, \mathrm{N}$-dimethylformamide), $0.12 \mathrm{mM} \mathrm{K}_{3} \mathrm{Fe}(\mathrm{CN})_{6}, 0.12 \mathrm{mM} \mathrm{K}_{4} \mathrm{Fe}(\mathrm{CN})_{6}$ and $1 \mathrm{mM} \mathrm{MgCl} / 2 \mathrm{PBS}$ (pH 6.0) at $37^{\circ} \mathrm{C}$ overnight in $\mathrm{CO}_{2}$-free incubator. The day after, cells were washed three times with deionized water and imaged with a Zeiss Axiovert $40 \mathrm{CFL}$ microscope using a $\times 10$ objective.

Cell proliferation. IMR90 $\left(4 \times 10^{4}\right)$ were plated in a 24 -well plate and placed onto the stage of the Incucyte Live Content Imaging instrument. Nine fields for each well were collected every 2 hours for the first day and then every hour for the following days. Cell proliferation was automatically determined from bright-field images by calculating cell confluence at different time points, using the appropriate function of the Incucyte software. Cell media was replaced daily with media supplemented with the indicated drugs.

Generation of the metabolic models of $\mathrm{Fh}^{\mathrm{L} / \boldsymbol{\Delta}}$ and $\mathrm{Fh} \mathrm{f}^{\mathrm{fl} / \mathrm{fl}}$ cells. To investigate the metabolic changes that occur due to FH-inactivation metabolic models of Fh ${ }^{4}$ $\Delta^{\Delta}$ and Fh1 $\mathrm{f}^{f / f l}$, cell lines were constructed based on their transcriptomics and uptake-secretion metabolomics. The construction was conducted in two stages. In the first stage, each model was constrained to obtain an optimal fit to the gene expression signature of the cell line by solving a Mixed Integer Linear Programming problem, via the integrative Metabolic Analysis Tool (iMAT) ${ }^{32,33}$. In the second stage, each model was further constrained to obtain an optimal fit to the measured uptake-secretion flux rates. The latter was achieved by forming the following problem:

$$
\min \sum_{i \in R_{\text {fux }}} y_{i}
$$

subject to

$$
\begin{gathered}
S \cdot v=0 \\
\forall i: v_{i, \text { min }} \leq v_{i} \leq v_{i, \max } \\
\forall i \in R_{m}:\left|v_{i}-f_{i}\right| \leq y_{i}
\end{gathered}
$$

Where $\mathbf{v}$ is the flux vector and $S$ is an $m \times n$ stoichiometric matrix, in which $m$ is the number of metabolites and $n$ is the number of reactions. The mass balance constraint is enforced by equation (2). Thermodynamic and enzyme capacity constraints are imposed in equation (3), by setting $v_{i, \min }$ and $v_{i, \max }$ as a lower and upper bound of the flux values through reaction $i$, respectively. In equation (4), $R_{m}$ includes the set of measured reactions; for each measured reaction $i$, the variables $y_{i}$ represents the deviation of its computed flux rate, denoted as $v_{i}$, from its measured flux rate, denoted as $f_{i}$. The objective function equation (1) is to minimize the deviation of the computational flux rates from the measured flux rates. To avoid the non-linear constraint imposed in equation (4) two variables, $y^{+}$and $y^{-}$, are 
introduced to convert the problem to its equivalent linear form.

$$
\min \sum_{i \in R_{\text {flux }}}\left(y_{i}^{+}+y_{i}^{-}\right)
$$

subject to

$$
\begin{gathered}
S \cdot v=0 \\
\forall i: v_{i, \min } \leq v_{i} \leq v_{i, \max } \\
\forall i \in R_{m}:-y_{i}^{-} \leq v_{i}-f_{i} \leq y_{i}^{+} \\
0 \leq y_{i}^{+}, y_{i}^{-}
\end{gathered}
$$

A solution found by the Mixed Integer Linear Programming solver to the iMAT problem and then by a Linear Programming solver to the problem formulated above is guaranteed to be optimal, meaning, to maximize the similarity to the expression and then, under the optimal fit to the transcriptomics to obtain a maximal fit to the flux measurements. However, the solution is not necessarily unique, as a space of alternative optimal solutions may exist. The space of optimal solutions represents various steady-state flux distributions attaining the same similarity with the expression data and flux measurements. To account for these alternative solutions, the optimal similarity to the expression and flux measurements is calculated. The model is then constrained to attain this optimal similarity, which reduces the solution space significantly.

To explore the optimal solution space of the model, that is, the feasible metabolic states which maximize the fit to the experimental data, flux variability analysis (FVA) ${ }^{34}$ was employed. FVA obtains for each reaction its minimal and maximal attainable flux, subject to the aforementioned constraints. In addition, by applying FVA, the maximal production rate of every metabolite in the models was computed; in this case, the production of a metabolite was defined as the sum of its production rates in all the different compartments of the model (for example, mitochondria, cytoplasm). For each metabolic pathway, the median fold-change in the production of its metabolites was computed based on the pathway definition of ref. 13. Fianlly, the redox potential of the models was estimated by computing their capacity to produce $\mathrm{NADH}$ and NADPH from $\mathrm{NAD}^{+} \mathrm{NADP}^{+}$, respectively.

The metabolic model of the FH-deficient cells were utilized to examine the dependency of GSH production on the uptake-secretion rates of different metabolites, including amino acids and main carbon sources. First, for each metabolite in this set, the feasible flux range of its exchange reaction was computed via FVA. An exchange reaction represents the secretion or uptake of a given metabolite. A positive flux rate through an exchange reaction denotes secretion and a negative flux rate denotes uptake. Next, for every one of the metabolites the model was constrained to carry out an increasing flux of its exchange reaction, which is within its feasible flux range. Under each of these constraints, the maximal production of reduced GSH in the model was computed. Finally, for each metabolite, the Spearman correlation between the upper bounds of the metabolite exchange reaction and the pertaining maximal production rates of GSH was computed. A positive correlation, as found between GSH production and arginine, denotes that a higher flux rate through the exchange reaction of the metaboliteless uptake or more secretion-enables the model to produce GSH more rapidly. Likewise, a negative correlation, as found between GSH production and cystine, denotes that a lower flux rate through the exchange reaction of the metabolitemore uptake or less secretion-enables the model to produce GSH more rapidly.

The ability of the models to predict the measured flux rates was examined in a leave-one-out cross-validation process. Every iteration, the models were regenerated based on their gene expression and a partial set of the metabolic uptake and secretion rates. These models were then used to compute via FVA the maximal and minimal flux rate of the exchange reaction whose flux measurement was omitted from the construction process. Significant correlation was found between the measured and predicted flux rates (Supplementary Table 2).

Following the validation of the models, they were utilized to systematically explore the metabolic differences between the Fh $1^{\Delta / \Delta}$ and Fh $1^{f l / f l}$ cells. The capacity of the two models to produce each of their 1,491 metabolites was computed. The FH-deficient model was found to have a higher capacity to produce 43 metabolites (Supplementary Fig. 1b). Among the top ones are four derivatives of GSH: Oxidized GSH, reduced GSH, (R)-S-Lactoylgutathione and S-Formylglutathione (Supplementary Fig. 1b). These results suggest that FH deficiency induces oxidative stress. To further examine this hypothesis, the ability of the Fh1 $1^{f / f l}$ and Fh1 $1^{\Delta / \Delta}$ models to produce reducing power in the form of NADH and NADPH was computed. Indeed, the Fh1 ${ }^{\text {fl/fl }}$ model was found to have a significantly higher capacity to produce NADH and NADPH, compared with the Fh1 $1^{\Delta / \Delta}$ model.

Following the discovery of GSH succination in the FH-deficient cells, the models were reconstructed with GSH succination (see next section). According to the updated models, GSH succination further severely limits NADPH and NADH production in Fh1 ${ }^{\Delta / \Delta}$ cells. This strong coupling between GSH succination and decreased NADH/NADPH production is highly notable, since the activity of almost all other reactions in the $F 1^{\Delta / \Delta}$ model does not entail such a decrease (Supplementary Fig. 1c, Wilcoxon ranksum $P$ value of $7.91 \mathrm{e}-04$ ). Further computational analysis revealed that this coupling arises since, in the Fh $1^{\Delta / \Delta}$ cells, GSH synthesis depends on cystine uptake, rather than on methionine uptake (Supplementary Fig. 3b). The synthesis of GSH from cystine causes the oxidation of two GSH molecules. As reducing them requires NADPH, it imposes a constraint to activate pathways that contribute to NADPH production.

GSH succination was introduced to the models by adding the following reactions

(1) Mitochondrial reaction: reduced GSH $[\mathrm{m}]+$ fumarate $[\mathrm{m}] \rightarrow$ succinicGSH

(2) Cytoplasmatic reaction: reduced GSH [c] + fumarate [c] $\rightarrow$ succinicGSH

(3) Sink reaction: succinicGSH

Reaction (3) denotes the secretion of succinicGSH. In the FH-deficient model, this reaction was constrained to carry a non-zero flux. Note that some of the succinicGSH may be metabolized via additional metabolic reactions which are yet to be discovered, as opposed to secreted. However, currently, the models cannot account for such reactions.

\section{References}

1. Tomlinson, I. P. M. et al. Germline mutations in FH predispose to dominantly inherited uterine fibroids, skin leiomyomata and papillary renal cell cancer. Nat. Genet. 30, 406-410 (2002).

2. Frezza, C., Pollard, P. J. \& Gottlieb, E. Inborn and acquired metabolic defects in cancer. J. Mol. Med. 89, 213-220 (2011).

3. Isaacs, J. S. et al. HIF overexpression correlates with biallelic loss of fumarate hydratase in renal cancer: novel role of fumarate in regulation of HIF stability. Cancer Cell 8, 143-153 (2005).

4. Pollard, P. J. et al. Accumulation of Krebs cycle intermediates and overexpression of HIFlalpha in tumours which result from germline $\mathrm{FH}$ and SDH mutations. Hum. Mol. Genet. 14, 2231-2239 (2005).

5. Adam, J. et al. Renal cyst formation in Fh1-deficient mice is independent of the Hif/Phd pathway: roles for fumarate in KEAP1 succination and Nrf2 signaling. Cancer Cell 20, 524-537 (2011).

6. Bardella, C. et al. Aberrant succination of proteins in fumarate hydratasedeficient mice and HLRCC patients is a robust biomarker of mutation status. J. Pathol. 225, 4-11 (2011).

7. Ooi, A. et al. An antioxidant response phenotype shared between hereditary and sporadic type 2 papillary renal cell carcinoma. Cancer Cell 20, 511-523 (2011).

8. Frezza, C. et al. Haem oxygenase is synthetically lethal with the tumour suppressor fumarate hydratase. Nature 477, 225-228 (2011).

9. Sudarshan, S. et al. Fumarate hydratase deficiency in renal cancer induces glycolytic addiction and hypoxia-inducible transcription factor 1alpha stabilization by glucose-dependent generation of reactive oxygen species. Mol. Cell. Biol. 29, 4080-4090 (2009).

10. Sullivan, L. B. et al. The proto-oncometabolite fumarate binds glutathione to amplify ROS-dependent signaling. Mol. Cell 51, 236-248 (2013).

11. Shlomi, T., Benyamini, T., Gottlieb, E., Sharan, R. \& Ruppin, E. Genome-scale metabolic modeling elucidates the role of proliferative adaptation in causing the Warburg effect. PLoS Comput. Biol. 7, e1002018 (2011).

12. Folger, O. et al. Predicting selective drug targets in cancer through metabolic networks. Mol. Syst. Biol. 7, 501 (2011).

13. Duarte, N. C. et al. Global reconstruction of the human metabolic network based on genomic and bibliomic data. Proc. Natl Acad. Sci. USA 104, 1777-1782 (2007).

14. Fabrini, R. et al. The extended catalysis of glutathione transferase. FEBS Lett. 585, 341-345 (2011).

15. Zheng, L. et al. Reversed argininosuccinate lyase activity in fumarate hydratasedeficient cancer cells. Cancer Metab. 1, 12 (2013).

16. Yang, Y. et al. UOK 262 cell line, fumarate hydratase deficient (FH-/FH-) hereditary leiomyomatosis renal cell carcinoma: in vitro and in vivo model of an aberrant energy metabolic pathway in human cancer. Cancer Genet. Cytogenet. 196, 45-55 (2010).

17. Ashrafian, H. et al. Expression profiling in progressive stages of fumaratehydratase deficiency: the contribution of metabolic changes to tumorigenesis. Cancer Res. 70, 9153-9165 (2010).

18. Serrano, M. \& Blasco, M. A. Putting the stress on senescence. Curr. Opin. Cell Biol. 13, 748-753 (2001).

19. Pollard, P. J. et al. Targeted inactivation of fhl causes proliferative renal cyst development and activation of the hypoxia pathway. Cancer Cell 11, 311-319 (2007).

20. Scholzen, T. \& Gerdes, J. The Ki-67 protein: from the known and the unknown J. Cell. Physiol. 182, 311-322 (2000).

21. Kaspar, J. W., Niture, S. K. \& Jaiswal, A. K. Nrf2:INrf2 (Keap1) signaling in oxidative stress. Free Radical Biol. Med. 47, 1304-1309 (2009).

22. Gottlieb, E. \& Tomlinson, I. P. Mitochondrial tumour suppressors: a genetic and biochemical update. Nat. Rev. Cancer 5, 857-866 (2005).

23. Young, A. P. et al. VHL loss actuates a HIF-independent senescence programme mediated by Rb and p400. Nat. Cell Biol. 10, 361-369 (2008).

24. Chen, Z. et al. Crucial role of p53-dependent cellular senescence in suppression of Pten-deficient tumorigenesis. Nature 436, 725-730 (2005). 
25. Collado, M., Blasco, M. A. \& Serrano, M. Cellular senescence in cancer and aging. Cell 130, 223-233 (2007).

26. Michaloglou, C. et al. BRAFE600-associated senescence-like cell cycle arrest of human naevi. Nature 436, 720-724 (2005).

27. Kaplon, J. et al. A key role for mitochondrial gatekeeper pyruvate dehydrogenase in oncogene-induced senescence. Nature 498, 109-112 (2013).

28. Ireland, H. et al. Inducible Cre-mediated control of gene expression in the murine gastrointestinal tract: effect of loss of beta-catenin. Gastroenterology 126, 1236-1246 (2004).

29. Deng, C., Zhang, P., Harper, J. W., Elledge, S. J. \& Leder, P. Mice lacking p21CIP1/WAF1 undergo normal development, but are defective in G1 checkpoint control. Cell 82, 675-684 (1995).

30. Ashburner, M. et al. Gene ontology: tool for the unification of biology. The Gene Ontology Consortium. Nat. Genet. 25, 25-29 (2000).

31. Mathew, R., Degenhardt, K., Haramaty, L., Karp, C. M. \& White, E. Immortalized mouse epithelial cell models to study the role of apoptosis in cancer. Methods Enzymol. 446, 77-106 (2008).

32. Zur, H., Ruppin, E. \& Shlomi, T. iMAT: an integrative metabolic analysis tool. Bioinformatics 26, 3140-3142 (2010).

33. Shlomi, T., Cabili, M. N., Herrgard, M. J., Palsson, B. O. \& Ruppin, E. Networkbased prediction of human tissue-specific metabolism. Nat. Biotechnol. 26, 1003-1010 (2008).

34. Mahadevan, R. \& Schilling, C. H. The effects of alternate optimal solutions in constraint-based genome-scale metabolic models. Metab. Eng. 5, 264-276 (2003).

\section{Acknowledgements}

This work was supported by Cancer Research UK. S.C. was partially funded by FEBS Long-Term Fellowship. L.J. is partially funded by the Edmond J. Safra bioinformatics centre and the Israeli Center of Research Excellence programme (I-CORE, Gene Regulation in Complex Human Disease Center No 41/11) and an Adams fellowship. E.R. research in cancer is supported by grants from the Israeli Science Foundation (ISF), the Israeli Cancer Research Fund (ICRF) and the I-CORE programme. We thank UOB
Tumor Cell Line Repository and Dr W. Marston Linehan, National Cancer Institute, Bethesda, MD, USA for providing us with the UOK262 cell lines.

\section{Author contributions}

E.G., C.F. and L.Z. designed the study and wrote the paper. C.F., L.Z., E.D.M. and S.C. performed the experiments on the Fh1-deficient models. M.S., T.I.J. and E.G. performed the experiments on the IMR90 cell lines. R.E.-E. and N.A.M. performed, respectively, the NMR and LC-MS/MS characterization of succinic GSH. A.H. and G.K. performed the bioinformatics analyses of the metabolomics of Fh1-deficient cells. K.B. supervised the in vivo experiments and A.K. and J.D.G.L performed the histopathological analyses of the kidney sections. L.J. and E.R. performed the metabolic modelling analyses. All authors have read and commented on the manuscript.

\section{Additional information}

Supplementary Information accompanies this paper at http://www.nature.com/ naturecommunications

Competing financial interests: The authors declare no competing financial interests.

Reprints and permission information is available online at http://npg.nature.com/ Reprintsandpermissions/

How to cite this article: Zheng, L. et al. Fumarate induces redox-dependent senescence by modifying glutathione metabolism. Nat. Commun. 6:6001 doi: 10.1038/ncomms7001 (2015).

\section{(c) (i)}

This work is licensed under a Creative Commons Attribution 4.0 International License. The images or other third party material in this article are included in the article's Creative Commons license, unless indicated otherwise in the credit line; if the material is not included under the Creative Commons license, users will need to obtain permission from the license holder to reproduce the material. To view a copy of this license, visit http://creativecommons.org/licenses/by/4.0/ 